\title{
Robustness and Performance Tradeoffs in Control Design for Flexible Structures
}

\author{
Gary J. Balas \\ Dept. of Aerospace Engineering and Mechanics \\ University of Minnesota \\ Minneapolis, MN 55455
}

\author{
John C. Doyle \\ Dept. of Electrical Engineering \\ California Institute of Technology \\ Pasadena, Ca. 91125
}

\begin{abstract}
Linear control design methodologies for flexible structures require the nominal structural model to be formulated as a linear, time invariant system. Although this model may describe the physical system accurately, any model is only an approximation of the physical system. There is always some uncertainty present even when the underlying process is essentially linear. This uncertainty may be due to incomplete knowledge of the physical paramcters, neglected high frequency dynamics or invalid assumptions made in the model formulation. Descriptions of these uncertainties determine the tradeoff between achievable performance and robustness of the control design.

This paper focuses on designing control laws for the Caltech flexible structure experiment using a nominal design model with varying levels of uncertainty. It is shown that an improper selection of nominal and uncertainty models, may lead to unstable or poor performing controllers on the actual system. In contrast, if descriptions of uncertainty are overly conservative, performance of the closed-loop system may be severely limited. Therefore, tight uncertainty bounds are required to provide robust control designs which achieve high performance when implemented on the actual system. Experimental results of control laws synthesized for different uncertainty levels on the Caltech structure are presented.
\end{abstract}

\section{Introduction}

Stringent requirements envisioned for the pointing and shape accuracy of future space missions necessitate advances in the control of large flexible structures. These structures will be extremely flexible, with little natural damping and modes which are densely packed throughout the frequency domain. Due to their size and complexity, ground testing of these structures in Earth's environment will lead to system models that are inaccurate for operation in a zero-gravity environment. Even with on-orbit identification of the structure, discrepancies between their mathematical models and the "real" structure will still exist, although to a lesser extent. Therefore, control design methods must be developed to account for model inaccuracies or uncertainties. Such methods should optimize the robustness and performance characteristics of control laws based on the accuracy of the design model.

Robust control design methods optimize control laws based on knowledge of how model error enters into the problem description. Since controller optimization is based on mathematical system descriptions, accurate accounting and characterization of variations between "real" flexible structures and their mathematical models is essential. These variations may be due to non-physically based assumptions, including neglected nonlinearities, unmodeled dynamics and errors associated with model parameters (e.g. mode shapes, natural frequencies, and damping values). Differences due to the first two assumptions can generally be approximated as a norm bounded, frequency domain error (referred to as unstructured uncertainty) when the nonlinearities are small. As the nonlinearities become more dominant, frequency domain descriptions of the nonlinearities yield poor models, and more intelligent means of modeling need to be employed.

Variations in the natural frequencies, damping values and mode shapes can be handled more systematically. These errors are highly structured and often cannot be treated as norm-bounded uncertainties without substantially increasing the conservativeness of the mod- els. This additional conservativeness can severely limit the performance of the control design. Variation in the model parameters is defined as parametric uncertainty. The combination of unstructured and parametric uncertainty leads to structured uncertainty in the problem formulation. Similarly, unstructured uncertainty at the component level results in structured uncertainty when viewed at the system level. Mathematical models of structured and unstructured uncertainties need to be included in the control problem formulation to optimize the desired performance objectives.

This research addresses incorporating model mismatch between the physical system and its mathematical descriptions into the control design process. Control design models of flexible structures are developed to fit into the structured singular value $(\mu)$ framework. The final design model is based on a nominal (base) plant model, uncertainty descriptions, and additive noise models, and defines a family or set of uncertain plant models in which the "real" system is assumed to reside. $\mu$-synthesis methods which integrate $\mu$-analysis and $H_{\infty}$ control design methods are applied to these model sets to generate control laws for the given system. The Caltech flexible structure experiment is used as a test bed in this research to validate results experimentally. Since the "real" structure is assumed to lie within the model set, measures of robustness and performance characteristics of the control laws can be evaluated and predicted when implemented on the flexible structure experiment.

The focus of this paper is on designing control laws for the Caltech flexible structure experiment by varying the level of uncertainty in the design model and applying them to the experimental structure. It is shown that a controller synthesized for a plant model, which is not described accurately by the nominal and uncertainty models, maybe unstable or exhibit poor performance when implemented on the actual system. It will be shown that similar performance, both theoretically and experimentally, was obtained for a surprisely wide range of uncertainty levels in the design model. This suggests that while it is important to have reasonable uncertainty models, it may not always be necessary to pin down precise levels (i.e. weights) of the uncertainty descriptions.

The following section describes the Caltech flexible structure experiment and formulation of the nominal structural model. Section 3 contains a brief overview of the structured singular value $(\mu), H_{\infty}$ control design, and $\mu$-synthesis design techniques. Tradeoffs associated with uncertainty modeling of flexible structures are discussed in Section 4. These tradeoffs are incorporated into the problem formulation in the form of robustness and performance measures. A series of controllers are synthesized based on different uncertainty descriptions. Section 5 discusses the experimental results of the implementation of the control designs. A summary of these results is presented in Section 6 along with a discussion of future research directions.

\section{Caltech Experimental Flexible Structure}

The Caltech experimental flexible structure is designed to include a number of attributes associated with large flexible space structures [Balas, BalDoy1, BalDoy2]. These include lightly damped, closely spaced modes, collocated and noncollocated sensors and actuators, and numerous modes in the controller crossover region. In addition to these considerations, expandability of the structure is a desired 


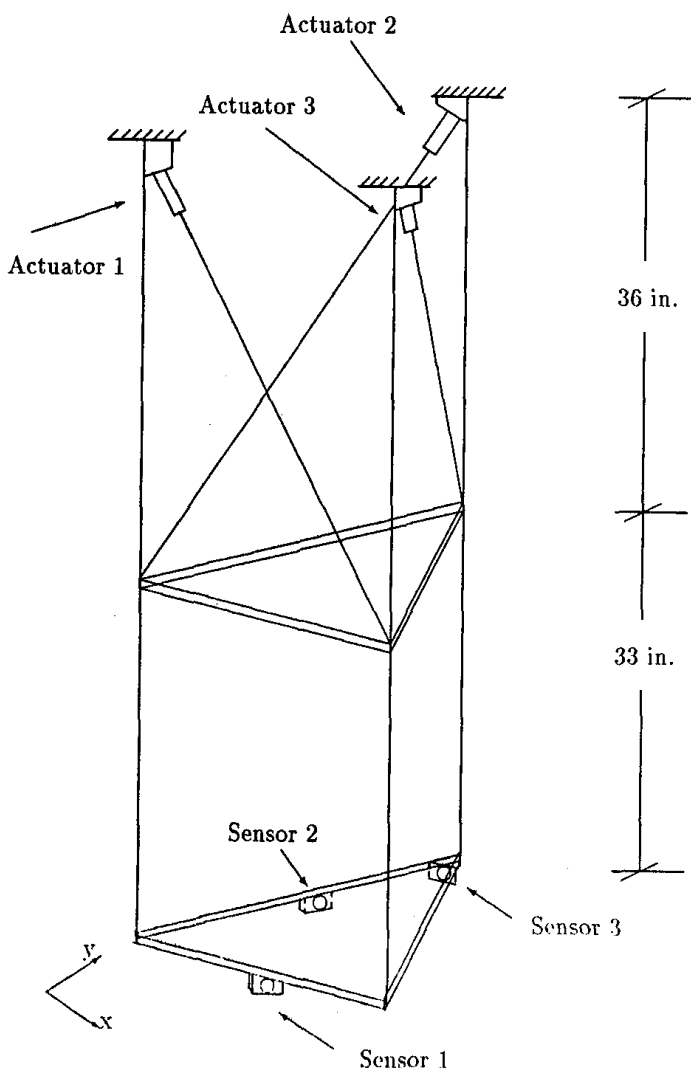

Figure 1: Phase I Caltech Flexible Structure

feature. Modular construction provides a means for increasing the modal density in a frequency range of interest.

The initial experimental structure, figure 1 , consists of two stories, three longerons (columns) and three noncollocated sensors and actuators. The first story columns are $.838 \mathrm{~m} \mathrm{(33} \mathrm{in.)} \mathrm{long,} 6.35 \mathrm{~mm}\left(\frac{1}{4} \mathrm{in}\right.$.) diameter aluminum rods. The second story columns measures $.914 \mathrm{~m}$ (36.0 in.) with $4.76 \mathrm{~mm}\left(\frac{3}{16} \mathrm{in}\right.$.) diameter aJuminum rods. Including the platforms, the structure has a height of $1.651 \mathrm{~m}$ ( $65 \mathrm{in}$.). The two platforms are the shape of an equilateral triangle with a $.406 \mathrm{~m}(16 \mathrm{in}$.) base. The longerons are connected between the stories via a triangular mating fixture and three bolts. This allows for the easy addition of stories to the structure. All the longerons are shrunk fit and welded to their mating brackets to reduce the effects of joint nonlinearities.

The first story platform is a $9.52 \mathrm{~mm}(3 / 8 \mathrm{in}$.) thick plate of aluminum, weighing $2.36 \mathrm{~kg}(5.2 \mathrm{lbs})$, with diagonal mounting brackets for attachment of the actuator diagonals. The second story platform is a $6.35 \mathrm{~mm}(1 / 4 \mathrm{in}$.) thick plate of aluminum with mounting holes for three accelerometers. It weighs $1.55 \mathrm{~kg}(3.4 \mathrm{lbs})$. A small offset mass is located on the second story platform to lower the torsional nat ural frequencies. The entire structure hangs from a moviting structure fixed to the ceiling to alleviate the problem of buckling of the longerons. The three actuators are attached to the mounting structure and act along the diagonals of the first story. The three sensors are accelerometers that are located on the second bay platform.

The two stories are designed to have the same first bending natural frequency. This is obtained by selecting the stiffness of the second story columns to be one fourth the stiffness of the first column with a similar ratio between the masses of the two stories. This ratio of stiffness and masses allows the interaction of the two stories to decrease the first bending natural frequency of the combined structure without significantly spreading out the remaining modes. The interaction leads to poor performance of collocated velocity feedback at the voice coil actuators. One can see that fixing the first story to be rigid (which is similar to implementating a collocated velocity feedback law at the actuators) provides little reduction in the second floor motion for similar second story excitation.

\subsection{Voice Coil Actuators}

The voice coil actuators are fabricated by Northern Magnetics Inc. They are similar to typical loudspeakers, outputting a force proportional to the input voltage. The actuators are mounted in line with the column diagonals and are rated at $\pm 1.36 \mathrm{~kg}(3 \mathrm{lbs})$ of force at \pm 5 volts. Command signals are supplied to each actuator through a current amplifier from a Masscomp computer used to implement real time control laws. The actuator model is derived from a theoretical model and bench tests. Static tests were run for input voltages between \pm 5 volts to formulate a linear relationship between the input voltage and output force. Frequency response data obtained from input/output experiments verified this relationship with the actuator bandwidth determined to be $60 \mathrm{~Hz}$.

\subsection{Air Actuators}

Three air actuators are used as input disturbances to the second story platform. Each actuator is placed to blow a stream of air directly on each sensor. These actuators meter compressed air lines, which are pulsed on and off by solenoids. Models of air actuators are difficult to formulate because no accurate measurement of the orifice diameter, air pressure in the line, or force being exerted at the sensors is available In an effort to develop a crude model, a sinusoidal frequency sweep between 1 and $6 \mathrm{~Hz}$ was input to the solenoid and the output response of the flexible structure was measured. The air actuators were found to have a flat frequency response up to approximately $4 \mathrm{~Hz}$ at which point their transfer function rolls off rapidly.

\subsection{Accelerometers}

Sunstrand QA-1400 accelerometers are used as the sensors. These accelerometers are mounted on the second story platform, located along the $x$-axis, $y$-axis, and at 45 degrees to both axes. The accelerometers have a flat frequency response between 0 and $200 \mathrm{~Hz}$ and are extremely sensitive. The noise associated with them is rated at $0.05 \%$ of the output at $0-10 \mathrm{~Hz}$ and $2 \%$ at $10-100 \mathrm{~Hz}$. Models of the accelerometers are generated from data supplied by Sunstrand. The sensors are scaled for accelerations of $.016 \mathrm{~g}$ per volt to provide a maximum \pm 5 volts output at peak accelerations of the input disturbance. Their output is conditioned by a $100 \mathrm{~Hz}$, fourth order Butterworth filter prior to being input into the Masscomp analog/digital (A/D) converter to provide attenuation of high frequency signals and noise.

Tests are performed to verify the noise characteristics of the accelerometer. Due to their sensitivity, the accelerometers are isolated from the building during testing by hanging them from a $3 \mathrm{ft}$. rubber band. Building vibration is two orders of magnitude higher than the sensor noise in the low frequency range and the accelerometer noise is in line with the manufacturer specifications.

\subsection{Modeling of Experimental Structure}

A model of the structure relating input signals to system outputs is desired for control design purposes. Initially, an input/output model is developed from first principles. This model of the structure is based 


\begin{tabular}{|c|c|c|c|c|}
\hline Mode & $\begin{array}{c}\text { NASTRAN } \\
\text { Natural } \\
\text { Frequency (Hz) }\end{array}$ & $\begin{array}{c}\text { Experimental } \\
\text { Natural } \\
\text { Frequency (Hz) }\end{array}$ & $\begin{array}{c}\text { Damping } \\
\text { Ratio }\end{array}$ & $\begin{array}{c}\text { Mode } \\
\text { Type }\end{array}$ \\
\hline 1 & .991 & 1.17 & $1.8 \%$ & $1 s t$ bending \\
2 & .992 & 1.19 & $1.8 \%$ & $1 s t$ bending \\
3 & 2.004 & 2.26 & $1.0 \%$ & $1 s t$ torsional \\
4 & 2.069 & 2.66 & $1.6 \%$ & $2 n d$ bending \\
5 & 2.100 & 2.75 & $1.8 \%$ & $2 n d$ bending \\
6 & 3.832 & 4.43 & $0.9 \%$ & $2 n d$ torsional \\
\hline
\end{tabular}

Table 1: Damping Ratios and Natural Frequencies of the Phase I Experiment

on the finite element method (FEM). Simply, the FEM approximates a distributed parameter system with an unlimited number of degrees of freedom by a discrete system with finite dimensionality. The finite element model provides a first approximation to the natural frequencies and mode shapes of the experiment.

The longerons are treated as space frame elements having three translational and three rotational degrees of freedom at each node and torsional and bending stiffnesses in two directions. The diagonals are circular bars that have the same bending stiffnesses in both directions. The longerons and diagonals are modeled as having fixed-fixed ends due to their welded end connections. All joints are modeled as rigid connections.

The accelerometers, mounting brackets, platforms and additional masses on the structure are modeled as lumped masses, with their inertia properties taken into account in the finite element description. When the control system is not activated, the diagonals in the first story ride on the bearings of the voice coil actuators. No force is exerted in the open-loop configuration. Hence, the voice coil actuators are modeled as having free axial motion and as fixed in the two transverse directions. In reality, the diagonals ride on bearings that exhibit some stiction, friction, and free play. The bearings cause the damping levels to vary with the excitation amplitude. These factors lead to errors between the finite element model and the experimentally derived transfer functions. The degrees of freedom associated with vertical motion (along the longerons) are neglected in the analysis, since they correspond to high frequency modes outside the bandwidth of the current control design objectives.

The first six global modes are of interest for control purposes. The first group of local modes, which involve bending of the longerons, occur in the frequency range of 37 to $43 \mathrm{~Hz}$. These local modes are accounted for in the control design to insure they are not destabilized. Attenuation of their vibration is not a performance criteria. Table 1 contains a list of natural frequencies derived from the Nastran finite element model compared with natural frequencies and damping derived from experimental data.

A multi-input/multi-output (MIMO) transfer function model is derived from the Nastran modal coefficients of the first six modes. There were significant errors noted between the FEM transfer function Bode plots and the experimental data. A comparison between the finite element model, using experimentally derived natural frequencies and damping levels, and experimentally derived transfer functions is shown in figures 2 and 3 . The variation in the natural frequencies was believed to be caused hy three phenomena: the wires connected to the accelerometer were tiglitly fixed to the columns and pulled taut, thereby adding stiffness to the longerons, the gravity load on the structure was neglected whicl leads to higher natural frequencies; and stiction/friction associated with the voice coil actuators was neglected, leading to nonlinear behavior.

\subsection{Identification of Experimental Transfer Functions}

System identificatioil techniques are used to develop more accurate multivariable descriptions of the structure for control design [BalDoy1, Balas]. First, Chebyshev polynomials are employed to fit phase I ex-
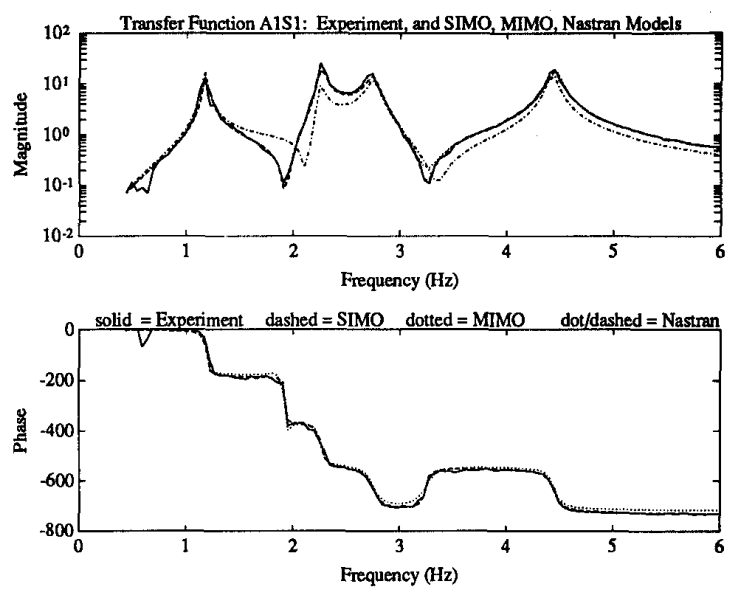

Figure 2: A Comparison Between the Experimental Data, Nastran and Identified Model for A1S1
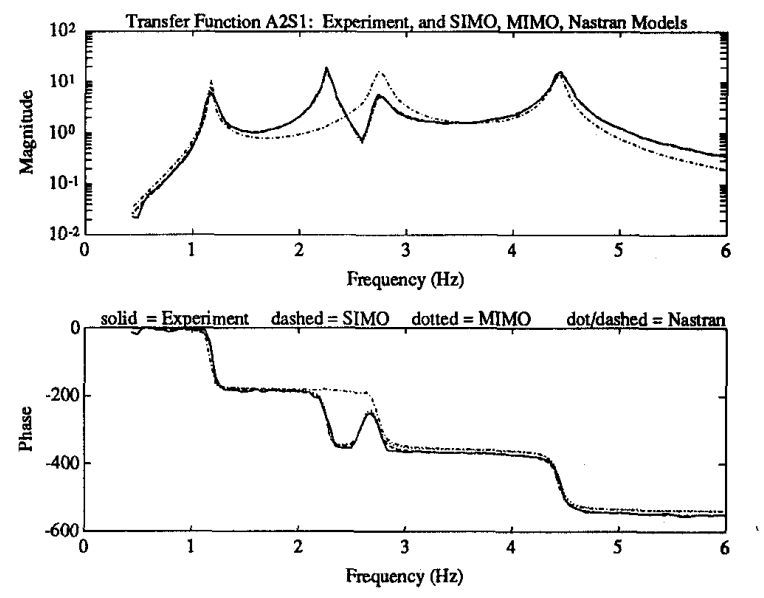

Figure 3: A Comparison Between the Experimental Data, Nastran and Identified Model for A2S1

perimental data with three single-input/multi-output (SIMO) transfer function models. The curve fitting technique uses a maximum magnitude error criteria to fit the data. This is similar to an $H_{\infty}$ norm bound on the error.

Combining these SIMO models leads to a multivariable model with 12 modes versus six in the original finite element model in the frequency range of interest. An ad hoc model reduction technique, based on a priori knowledge of the structural system and singular value decomposition methods, is used to develop a multivariable system description with six modes. Variations between the identified multivariable model and the experimental data is accounted for by several uncertainty descriptions. A comparison of the identified model, Nastran model and experimental data is shown in figures 2 and 3 . The identified multivariable model is used as the baseline nominal plant description in the control problem formulation. A more detailed description of the identification method can be found in [BalDoy1].

\subsection{Real Time Control Implementation}

The control designs are implemented on the Caltech flexible structure via a 5400 Masscomp computer. The real time control program implements a 3 input $/ 3$ output control law at $200 \mathrm{~Hz}$ and generates disturbance commands for the 3 air actuators. At $200 \mathrm{~Hz}$, a $60^{\text {th }}$ order control law, in modal coordinates, can be implemented. The 
system has a 12 bit A/D converter with a range of \pm 5 volts, .00244 volts per bit, and a 12 bit $D / A$ converter with a range of \pm 5 volts. The noise associated with the computer is \pm 1 lsb (least significant bit). The Masscomp computer is entirely dedicated to the closed-loop experiment during real time implementation of the control law.

A block diagram of the phase I experimental setup is shown in figure 4. The air actuators are used to excite the structure, causing the first and second story platform to vibrate. This, in turn, generates accelerations that are measured by the sensors. The sensor signals are filtered by a $100 \mathrm{~Hz}$, fourth order Butterworth filter and are then input to the Masscomp via the $\mathrm{A} / \mathrm{D}$ board. The control algorithm operates on these signals and generates force commands for the actuators, which are transmitted to the actuator via the $\mathrm{D} / \mathrm{A}$ converter. These signals, in the form of voltage levels, are input to the current amplifiers, which drive the voice coil actuators. Both the actuator command, which is trying to suppress the vibration, and the disturbance excitation affect the vibration of the second story platform. This cycle is continued throughout the implementation of the closed-loop experiment.

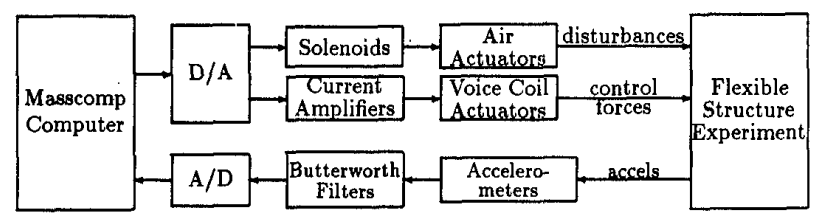

Figure 4: Block Diagram of Experimental Setup

\section{Structured Singular Value $(\mu)$ Framework}

This section briefly reviews frequency domain methods for analyzing the performance and robustness properties of feedback systems using the structured singular value $(\mu)$ [Doy1, Doy2, Pack, MUSYN]. The general framework, shown in figure 5 , is based on linear fractional transformations (LFTs). Any linear interconnection of inputs, outputs, and commands along with perturbations and a controller can be viewed in this context and rearranged to match this diagram. $P$ represents the system interconnection structure, $\Delta$ the uncertainties, and $K$ the control law. $v$ is a vector of exogenous inputs and disturbances, $e$ is a vector of errors to be kept small, $y$ is a vector of measurement

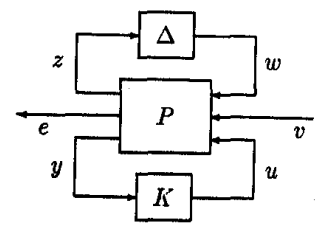

Figure 5: General Interconnection Structure
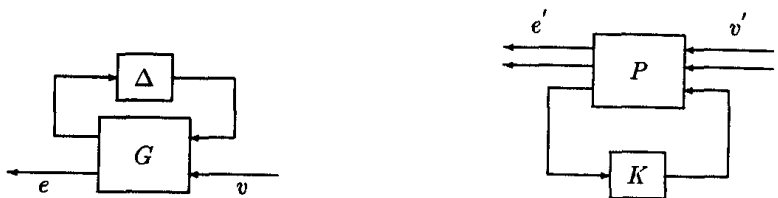

Figure 6: (a) Analysis

and

(b) Synthesis Problem

signals provided to the control design, $u$ is a vector of inputs from the control law, $z$ and $w$ are outputs to and from the uncertainty block.

\subsection{Definitions}

Following is a list of terms used extensively in this paper.

Nominal Stability (NS) The nominal plant model has to be stiali lized by the controller design.

Nominal Performance (NP) In addition to nominal stability, thir: nominal closed-loop response should satisfy some performanc: requirements. In the synthesis problem, performance is drefincol in terms of the weighted $H_{\infty}$-norms for the closed-loop systom transfer function between the exogenous inputs (disturbances) and "errors" (sensor outputs). This norm describes the "worstcase" closed-loop response, over frequency, to disturbances.

Robust Stability (RS) The closed-loop system must remain stable for all possible plants as defined by the uncertainty descriptions.

Robust Performance (RP) The closed-loop system must satisfy the performance requirement for all possible plants as defined by the uncertainty description.

The goal of a control design is to achieve robust performance when implemented on the physical system. One method that directly addresses the robust performance question, in a multivariable framework, is $\mu$-based analysis and synthesis techniques.

\subsection{Analysis Overview}

For the purpose of analysis, the controller may be represented as another system component. The inclusion of the controller into the plant reduces the diagram in figure 5 to that in figure 6-(a). The analysis problem involves determining whether the error $e$ remains in a desired set for sets of input $v$ and perturbation $\Delta$. The uncertainty in $v$ and $\Delta$ as well as the performance specifications on $e$ are normalized to 1 . This requires that all weighting functions and scalings be absorbed into the interconnection structure $G$. Furthermore, $G$ can be partitioned so that the input-output map from $v$ to $\epsilon$ can be expressed as the following linear fractional transformation:

$$
e=F_{u}(G, \Delta) v
$$

where

$F_{u}(G, \Delta)=G_{22}+G_{21} \Delta\left(I-G_{11} \Delta\right)^{-1} G_{12} ;$ $G=\left[\begin{array}{ll}G_{11} & G_{12} \\ G_{21} & G_{22}\end{array}\right]$

The nominal perfornance objective is simply

$$
\left\|G_{22}\right\|_{\infty}=\sup _{\omega} \bar{\sigma}\left(G_{22}(j w)\right) .
$$

Robust stability for unstructured uncertainty (only $\bar{\sigma}(\Delta) \leq 1$ known) depends on $\left\|G_{11}\right\|_{\infty}$. Unfortunately, the infinity norm measure of robust stability is often overly conservative when dealing with robust performance and realistic models of plant uncertainty. To handle these questions, a more complicated mathematical object, the structured singular value $(\mu)$, is required.

First assume that $\Delta$ has structure and belongs to the set

$$
\Delta=\operatorname{diag}\left(\Delta_{1}, \Delta_{2}, \ldots, \Delta_{n}\right) \text { and } B \underline{\Delta}=\{\Delta \in \underline{\Delta} \mid \bar{\sigma}(\Delta) \leq 1\}
$$

For a complex matrix, $M \in C^{n \times n}$, and for simplicity, restricting the uncertainty to full block, $\mu_{\Delta}(M)$ is defined as

$$
\mu_{\Delta}(M):=\min \frac{1}{\{\bar{\sigma}(\Delta: \Delta \in \underline{\operatorname{det}}(I+M \Delta)=0)\}}
$$

and has the property $\mu(\alpha M)=|\alpha| \mu(M)$. Obviously, $\mu$ is a function of $M$ which depends on the structure of $\underline{\Delta}$. For this discussion, the structure should be clear from the context. Let

$$
\begin{aligned}
& \underline{U}=\left\{\operatorname{diag}\left(U_{1}, U_{2}, \ldots, U_{n}\right) \mid U_{i}^{*} U_{i}=I\right\} \\
& \underline{D}=\left\{\operatorname{diag}\left(d_{1} I, d_{2} I, \ldots, d_{n} I\right) \mid d_{i} \in \mathbb{R}_{+}\right\}
\end{aligned}
$$

where the set $\underline{U}$ and $\underline{D}$ match the structure of $\Delta$. Note that $\underline{U}$ and 
$\underline{D}$ leave $\Delta$ invariant in the sense that $\Delta \in \underline{\Delta}$ and $D \in \underline{D}$ implies that $\bar{\sigma}(\Delta U)=\bar{\sigma}(U \Delta)$ ard $D \Delta D^{-1}=\Delta$. The sets $\underline{U}$ and $\underline{D}$ can be used to obtain the bounds

$$
\sup _{U \in \underline{U}} \rho(M U) \leq \mu(M) \leq \inf _{D \in \underline{D}} \bar{\sigma}\left(D M D^{-1}\right)
$$

where $\rho$ denotes the spectral radius and $\bar{\sigma}$ denotes the maximum singular value.

Key theorems regarding $\mu$ prove that the lower bound is always an equality and the upper bound is an equality for $n \leq 3$ [Doy]. Unfortunately, the optimization problem implied by the lower bound has multiple local maxima and therefore does not immediately yield a reliable computational approach. Although $\bar{\sigma}\left(D M D^{-1}\right)$ is convex in $\ln (D)$ (so that the infimum can be found by searching over $n-1$ real parameters), the infimum is not necessarily equal to $\mu$. On the other hand, extensive experimentation [Doy2] indicates that the upper bound is close to $\mu$ in general. This result is simply a conjecture and hasn't been proven yet. The worst case ratio of the lower bound to the upper bound found to date is on the order of 0.85 .

The importance of $\mu$ for studying robustness of feedback systems is due to the following two theorems, which characterize the robust stability and robust performance of a system in the presence of structured uncertainty in terms of $\mu$.

Theorem: Rubust Stability (R.S.)

$$
F_{u}(G, \Delta) \text { stable } \forall \Delta \in B \Delta \text { iff } \sup _{\omega} \mu\left(G_{11}(j w)\right) \leq 1
$$

Theorem: Robust Performance (R.P.)

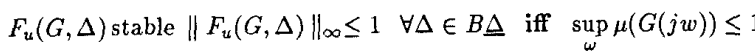

(where $\mu$ in Theorem R.P. is computed with respect to the structure $\left.\Delta=\left\{\operatorname{diag}\left(\Delta, \Delta_{n+1}\right)\right\}, \Delta \in \underline{\Delta}.\right)$ and

$$
\left\|F_{u}(G, \Delta)\right\|_{\infty} \equiv\left\|G_{11}+G_{12} \Delta\left(I-G_{22} \Delta\right)^{-1} G_{21}\right\|_{\infty}
$$

In words, robust performance of the closed-loop system $G$ is a $\mu$ test, across frequency, for the given block structure $\Delta$.

\subsection{Synthesis - $H_{\infty}$ Optimization}

For the purpose of synthesis, the $\Delta$ can be normalized to 1 and the normalizing factor absorbed into $P$. This results in the synthesis problem as shown in figure 6-(b). Hence, the synthesis problem involves finding a stabilizing controller $K$ such that the performance requirements are satisfied under prescribed uncertainties. The interconnection structure $P$ is partitioned such that the input-output map from $v^{\prime}$ to $e^{\prime}$ is expressed as the following linear fractional transformation

$$
e^{\prime}=F_{l}(P, K) v^{\prime}
$$

where

$$
F_{l}(P, K)=P_{11}+P_{12} K\left(I-P_{22} K\right)^{-1} P_{21} .
$$

For the $H_{\infty}$ optimal control problem, the objective is to find a stabilizing controller $K$ which minimizes $\left\|F_{l}(P, K)\right\|_{\infty}$. A detailed review of $H_{\infty}$ is given in [Francis] and state-space results are discussed in [DGKF, GlovDoy1, GlovDoy2].

\section{4 $\mu$-Synthesis Methodology}

The $\mu$-synthesis methodology is formulated to design controllers directly for robust performance. This technique integrates two powerful theories, $H_{\infty}$ optimization methods for synthesis and the structured singular value $(\mu)$ for analysis, into a systematic control design methodolgy.

Recall that an upper bound for $\mu$ may be obtained by scaling and applying the $\|\cdot\|_{\infty}$. Extending this concept to synthesis, the problem of robust controller design becomes that of finding a stabilizing controller $K$ and scaling matrix $D$ such that the quantity

$$
\left\|D F_{l}(P, K) D^{-1}\right\|_{\infty}
$$

is minimized. One approach for solving this problem is that of alternately minimizing the above expression for either $K$ or $D$ while holding the other constant, termed $D-K$ iteration. For fixed $D$, the controller synthesis is an $H_{\infty}$ optimization problem and can be solved using the well-known state-space method. With fixed $K$, the above quantity can be minimized at each frequency as a convex optimization in $\ln (D)$. The resulting data of $\mathrm{D}$ can be fit with an invertible, stable, minimum-phase, real-rational transfer function and wrapped back into the nominal interconnection structure. This process is carried out iteratively until a satisfactory controller is constructed.

The main objection to the above synthesis approach is that the $D-K$ iteration is not guaranteed to converge to a global minimum, even though each of the subproblems has global convergence. In spite of this, $\mu$-synthesis techniques seem to work well and have been used extensively in the area of designing robust control laws for flexible structures. Control designs using this methodology have been formulated for the vibration attenuation problem and implemented on a number of flexible structures experiments [Balas, BalChuD, BalDoy2]. The control designs synthesized using $\mu$-synthesis achieved a high level of vibration attenuation combined with good robustness characteristics. This technique is employed to address the tradeoffs between robustness and performance in the presence of structured uncertainty descriptions.

\section{Robustness and Performance Tradeoffs}

The selection of uncertainty descriptions plays a major role in the tradeoff between robustness and performance requirements in the con-

trol design process. A control law design based on an assumed "perfect" model leads to a high performance design on the model. However, when implemented on the "real" system, this controller may be destabilizing or exhibit poor performance. The destabilization or poor performance is attributed to the fact that the control design methodology optimizes the control law based only on the information provided, which is assumed to be "perfect." Models, though, are only approximations to "real" systems. Uncertainty descriptions are introduced to account for variations between these models and the physical system and provide a quantitative measure of their differences. It is essential that a control design methodology include uncertainty descriptions into the optimization process. The major benefit of $\mu$-synthesis techniques is the incorporation of both robustness and performance goals into a compatible design framework.

Selection of uncertainty descriptions and levels are not arbitrary. These are directly related to physical modeling of the problem. Descriptions of model error need to be developed based on the actual system characteristics. For example, choosing a large uncertainty model, unmotivated by the physical data, can lead to overly conservative control designs, thereby limiting performance of the control design. A tradeoff exists between robustness of the control law and performance objectives in the design process.

This section investigates this tradeoff in the selection of uncertainty descriptions and levels for the Caltech Phase I flexible structure experiment. Results indicate that an accurate plant (nominal) model and accurate uncertainty descriptions lead to control laws which exhibit superior performance when implemented or the physical system. Additionally, the selection of input or output uncertainty models to account for model errors has a direct bearing on the performance of multivariable control designs. This is contrast to single-input/singleoutput uncertainty models, which are not depended on the location of the uncertainty in the problem formulation.

A series of control laws are designed for the flexible structure experiment by varying the level of uncertainty and sensor noise weights. One set of control laws is designed using only an additive sensor noise model to account for uncertainty. These control laws destabilize the physical system until the sensor noise level model is increased in the problem formulatior to the magnitude of the flexible modes response. As the noise level is increased, the resulting control gain is reduced, limiting the closed-loop performance. The only control design which is stable when implemented is synthesized with a high level of sensor 
noise and provides little performance improvement compared to the open-loop response.

A second set of control laws is formulated using frequency domain uncertainty descriptions of the variations between the model and the "real" system. These designs make use of an additive uncertainty model to account for high frequency unmodeled dynamics and multiplicative input/output uncertainty to account for actuator/sensor errors and mode shape mismatch. As one traverses from a control law designed with only an additive uncertainty model to one with a significant amount of input or output uncertainty, the performance achieved on the experimental structure attains a maximum somewhere between these two extremes. This result clearly indicates the tradeoffs between robustness and performance in control design and the importance of uncertainty descriptions in the control design process.

\subsection{Control Objectives}

The control objective is to attenuate vibration of the first six natural frequencies in the Phase I Caltech flexible structure at the three accelerometer locations. These modes are between 1.2 and $4.5 \mathrm{~Hz}$ and consist of two first bending, two torsional and two second bending modes. The air disturbance, a sine sweep between 1 and $6 \mathrm{~Hz}$, enters the structure via air actuator 1, and blows directly on sensor 1 . The performance criteria is to minimize the maximum frequency response of the first six modes at the sensor locations as compared to the open-loop response for a worst case input signal. This specification is formulated as minimizing the $\|\cdot\|_{\infty}$ norm between the input disturbances and sensor outputs.

\subsection{Uncertainty Descriptions}

Frequency domain uncertainty descriptions are employed to account for the variation between the model and the "real" system. An additive uncertainty weight is used to account for the low frequency inaccuracies (below $0.5 \mathrm{~Hz}$ ) and the unmodeled high frequency dynamics (above $10 \mathrm{~Hz}$ ). The magnitude of the additive uncertainty weight at high frequency is selected to envelope the unmodeled modes of the system. The additive uncertainty weight assures that the high frequency modes are gain stabilized by requiring the control design to satisfy $\left\|W_{\text {add }}^{-1} K S\right\|_{\infty}<1$, where $K$ is the controller and $S$ is the sensitivity transfer function $\left(I-P_{n o m} K\right)^{-1}$. A plot of the frequency response of transfer functions between voice coil (VC) actuator 2 and the three sensors along with the additive uncertainty weight is shown in figure 7 . The additive uncertainty weight is given by

$$
W_{a d d}=8 \frac{(s+6)(s+12)(s+24)}{(s+.6)(s+400)^{2}}
$$

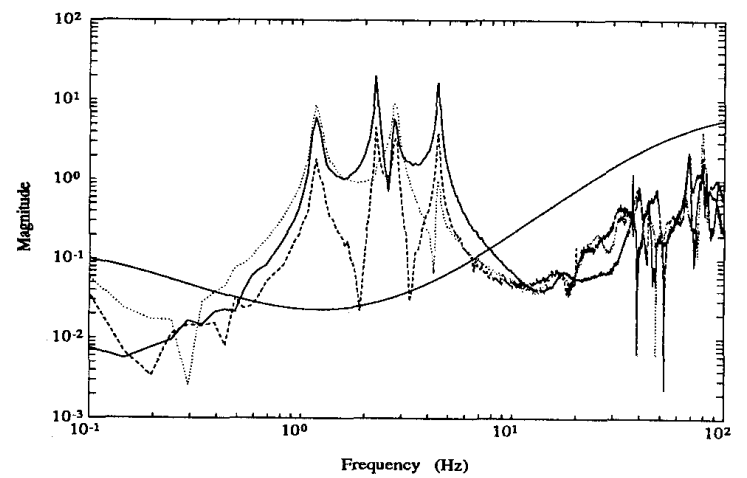

Figure 7: Frequency Response of Actuator 2 to Sensors and the Additive Uncertainty Weight

Within th: controller bandwidth, 1 to $5 \mathrm{~Hz}$, the additive uncertainty takes on a minimal value. This weight is purposely reduced within the frequency range performance to demonstrate how additional uncertainty descriptions, (i.e., multiplicative input and output uncer tainty weights), affect performance of the control designs. 'I'twr man nitude of the additive uncertainty weight is selerted to insuse lihat all control laws synthesized with this weight stabilize the structure. ('on. trol laws based on additive sensor noise models only do not includ th this weight leading to destabilizing control laws. The multiplicative uncers tainty on the inputs and outputs is varied independently betwe(e) (1) and $25 \%$ to gauge the effect on the robustness and performance propert tink of the control designs. These uncertainties are selected to be constant across frequency, since there is negligible frequency variation in the cr. rors between 1 and $5 \mathrm{~Hz}$. The additive uncertainty weight dominaten the uncertainty models outside this range. In the control problem formulation, the multiplicative weights are distributed between the inputs and outputs of the uncertainty blocks to provide better initial scaling for the $H_{\infty}$ control design algorithms.

\subsection{Control Problem Formulation}

The identified three-input/three-output nominal model of the flexible structure, $P_{\text {nom }}$, is used to describe the flexible structure experiment. It serves as a baseline model to which urcertainty models are appended. A block diagram of the problem formulation is shown in figure 8. As stated, the control design must be robust to unmodeled high frequency dynamics and model errors while attenuating the vibrational responses of the first six flexible modes. The additive uncertainty weight accounts for the neglected high frequency modes and some low frequency error. It is modeled as an unstructured full block uncertainty, $\Delta_{1}$, around the flexible structure model as seen in the block diagram. The additive uncertainty weight remains unchanged throughout the set of control designs.

Multiplicative input and output weights, actu and sensu (see figure 8), are the parameters varied to examine tradeoffs between the robustness and performance of the control designs. A constant input uncertainty, actu, is selected to account for actuator errors and mismatch between the input mode shapes and the experimental data. actu is varied from 0 to 0.5 , representing a 0 to $25 \%$ variation in the uncertainty level associated with the input signals to the flexible structure model. sensu represents a constant multiplicative output uncertainty. Sensor errors and output mode shape discrepancies are accounted for by this uncertainty description. One set of control laws is formulated with no output multiplicative uncertainty, sensu, and the input uncertainty, actu, varied. These control laws investigate the effect of input uncertainty descriptions on the performazce characteristics of the control designs when implemented on the physical system. Similarly, a set of control laws are synthesized with no input uncertainty, $a c t u$, and the output multiplicative uncertainty, sensu, varied between 0 and $0.5(0-25 \%$ uncertainty). The effect of output uncertainty on control designs is examined in this set of experiments.

The input and output multiplicative uncertainty models are described by full block unstructured uncertainty. Full block uncertainty descriptions indicate that cross coupling between the input (output) channels is allowed. The full block uncertainty description primarily accounts for mode shape errors that are present in the model. Scalar blocks restrict the uncertainty to the individual channels (i.e. no cross coupling of uncertainty). During the analysis stage of the control designs, comparisons are made between full and scalar block multiplicative uncertainty models. The three structured scalar uncertainty blocks have values of $\mu$ that are $1-3 \%$ less than the full block uncertainties. This differnce in $\mu$ values implies that if the structured uncertainty is a more accurate description of the physical system, it would have $1-3 \%$ better robustness margins and exhibit $1-3 \%$ better performance than the unstructured uncertainties when implemented. This is a modest difference, hence the unstructured uncertainties are used. The advantage of describing the input uncertainty by a full block is two fold: it reduces the number of uncertainty block in the $\mu$ analysis problem and the full block uncertainty accounts for cross feed between channels leading to a more robust control design. Output multiplicative uncertainty is also treated as a full block uncertainty. This uncertainty description accounts for output mode shape mismatch and crosstalk between the accelerometer signals. There is little difference 


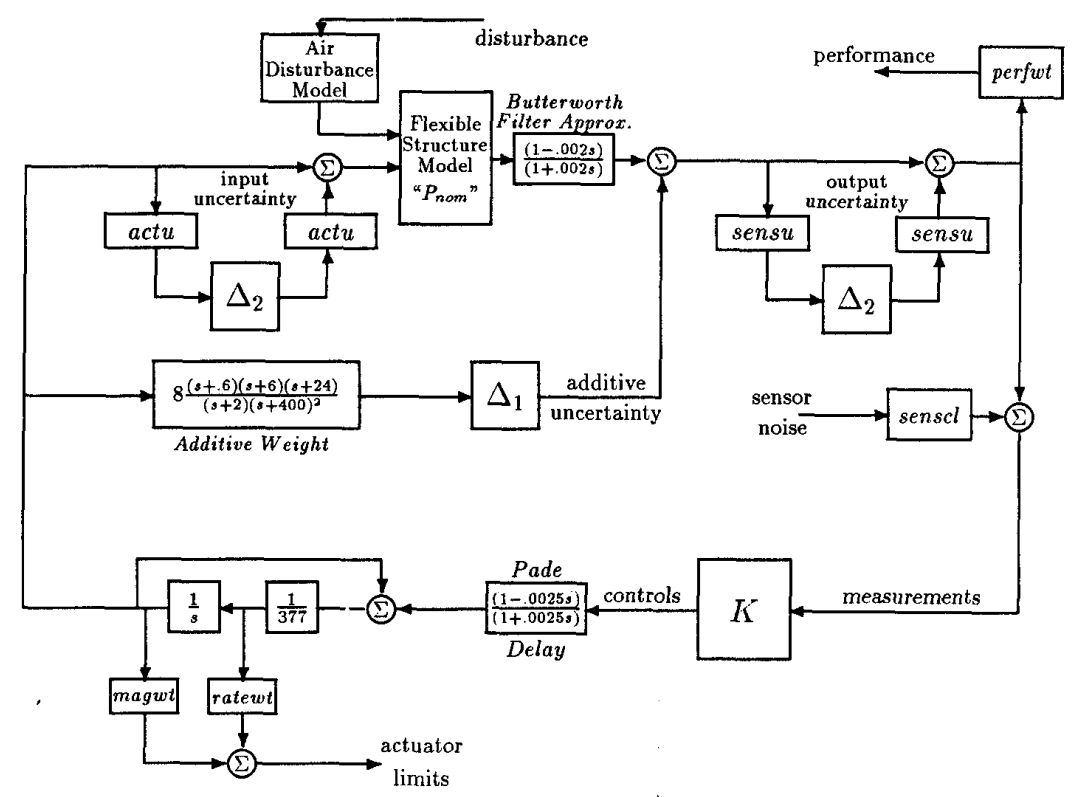

Figure 8: Block Diagram of Tradeoff Control Problem Formulation

(on the order of $1-3 \%$ ) whether a full block uncertainty or three scalar uncertainties are used in the analysis of the control laws with output multiplicative uncertainty. Hence, the multiplicative uncertainties are treated as one full block uncertainty.

There are a number of performance specifications associated with the experimental flexible structure. The performance objective is to minimize the maximum transfer function frequency response between the input disturbance and the three accelerometer outputs. The frequency range of interest is between 1 and $5 \mathrm{Izz}$, and contains the six flexible modes. To achieve this objective, the performance weight for vibration attenuation is selected as a constant scaling, perfwt, on the sensor outputs. The disturbance to acceleration output transfer functions are first normalized to 1 , then the performance weight, perfwt, is used to determine the amount of attenuation of the frequency domain peaks. A constant weighting is sufficient only if one desires the closed-loop performance transfer functions to be flat across frequency with no additional frequency shaping. Since the magnitude of the six flexible modes betwcen 1 and $5 \mathrm{~Hz}$ are all on the same order, a constant scaling provides a good performance objective and does not add additional states to the control problem.

The input disturbance enters via air actuator 1 and blows directly on sensor 1. A simple model of the excitation and the air actuators is included in the control design, $\left(\frac{10}{s+10}\right)$. The VC actuators are limited to $\pm 3 \mathrm{lbs}$ of force at $\pm 5 \mathrm{~V}$ with a $60 \mathrm{~Hz}$ rate limit. The actuator force limit is included in the control design by scaling the output magut to 1 when the force is at $\pm 3 l b s$. This scaling needs to be consistent with a unit input level of disturbance. Similarly, the $60 \mathrm{~Hz}$ rate limit is scaled with ratewt. The sensor noise level for the accelerometers is included as a performance limitation in the problem formulation. The weighting, senscl, is selected to be $2 \times 10^{-3}$ and represents an accelerometer signal to noise ratio of 250 . These performance specifications and limitations are accounted for in the $\mu$-framework by a full block unstructured uncertainty, resulting in a $\|\cdot\|_{\infty}$ norm measure. All performance requirements are satisfied when the $\|\cdot\|_{\infty}$ of the performance block is less than 1.

The accelerometers are filtered by $100 \mathrm{~Hz}$, fourth order Butterworth filters before being input into the Masscomp A/D channels. One can account for these filters with accurate fourth order models in each channel, but this would entail an additional 12 states in the problem formulation. A first order approximation of the filters, $\left(\frac{1-.002 s}{1+.002 s}\right)$ is used instead, reducing the additional states to 3 . This is an accurate approximation up to $40 \mathrm{~Hz}$ and accounts for the phase lag due to the filters. The first order Butterworth filter models are accurate far above the controller bandwidth, $5 \mathrm{~Hz}$, and any error induced by their approximation is accounted for by the additive uncertainty weights. A first order Pade approximation, $\left(\frac{1-.0025 s}{1+0025 s}\right)$, is included to model the $5 m s$ sample time delay associated with the Masscomp D/A channels. The complete block diagram is shown in figure 8 .

The block diagram is reformulated into the LFT general framework to design control laws using the $\mu$-synthesis methodology. A diagram of the LFT is shown in figure 9. The dimensions of the $\Delta$ blocks are: $3 \times 3$ for $\Delta_{1}, 3 \times 3$ for $\Delta_{2}$, and $6 \times 4$ for $\Delta_{3} . \Delta_{1}$ is associated with the additive uncertainty, $\Delta_{2}$ with the multiplicative input (output) uncertainty, and $\Delta_{3}$ is the performance block. All the $\Delta_{i}$ blocks are full blocks. Either input or output multiplicative uncertainty is included in the control problem formulation. In this set of designs, input and output uncertainty are not included simultaneously. The control design problem has structure due to the two uncertainty blocks and one performance block. A pure $H_{\infty}$ control design would synthesize a control law for one full block of size $12 \times 10$, neglecting the inherent structure associated with the three blocks. Ignoring the structure of the uncertainty block leads to overly conservative control laws. The $\mu$-synthesis methodology incorporates knowledge of this structure in the control design process, reducing the conservatism.

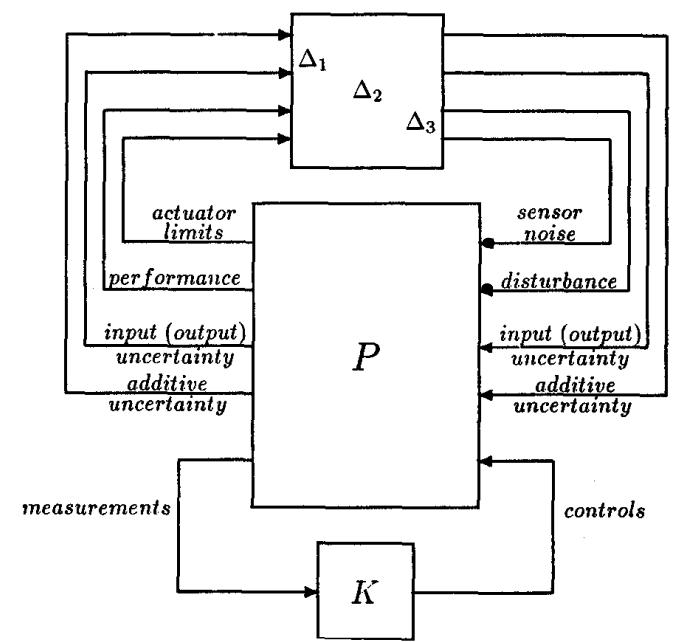

Figure 9: LFT of Tradeoff Control Problem Formulation 


\section{Control Designs}

\subsection{Sensor Noise Only}

Six control laws are synthesized based on the block diagram in figure 8 with no additive or multiplicative input/output uncertainty, (i.e., actu and sensu are set to zero). The sensor noise weight, senscl, is varied between $4 \times 10^{-3}$ and 2.3 to account for uncertainty and provide robustness in the control designs. Table 2 contains a list of the control law parameters used in the design and the results of implementation on the flexible structure experiment. The list includes the level of sensor noise, senscl, the performance scaling, perfwt, the value of $\mu$ achieved in the design and performance on the structure. Each control design is synthesized to achieve a $\mu$ value of 1 indicating that robust performance of the controller is achieved.

\begin{tabular}{|c|c|c|c|c|c|}
\hline Controller & senscl & perfwt & $\mu$ & $\begin{array}{c}\text { Predicted } \\
\text { Performance }\end{array}$ & $\begin{array}{c}\text { Experimental } \\
\text { Performance }\end{array}$ \\
\hline$K 1 s n$ & $4 \times 10^{-3}$ & 15.00 & 0.99 & .067 & Unstable \\
$K 2 s n$ & $4 \times 10^{-2}$ & 14.00 & 0.98 & .071 & Unstable \\
$K 3 s n$ & $4 \times 10^{-1}$ & 8.00 & 1.00 & .125 & Unstable \\
$K 4 s n$ & $9 \times 10^{-1}$ & 4.75 & 1.00 & .211 & Unstable \\
$K 5 s n$ & 1.15 & 4.75 & 1.00 & .253 & Unstable \\
$K 6 s n$ & 2.30 & 2.12 & 0.99 & .472 & 0.87 \\
\hline
\end{tabular}

Table 2: Parameters for Control Design with Sensor Noise

Figure 14 contain a singular value plot of the loop gain, $P_{\text {nom }} K$, for control laws $K 1 s n$. Controllers $K 1 s n$ through $K 5 s n$ are destabilizing when implemented on the experimental structure. This destabilization is due to the excessive gain of the control laws at high frequency, which destabilizes the unmodeled high frequency dynamics. One can see the large loop gain at high frequency associated with $K 1 s n$ in figure 14. The level of the sensor noise was raised to the magnitude of the flexible mode peaks in controller $K 6 s n$, leading to a reduction in the controller gain at low and high frequency. This stabilizes the system and thus reduces the performance of the control laws. Control laws $K 1 s n$ through $K 6 s n$ are stable and achieved their predicted performance in simulations using the nominal model.

Variation of the sensor noise level is an extreme example of the shortcomings associated with designing control laws based solely on additive noise models to account for model errors. It illustrates the need to provide information in the model formulation as to the fidelity of the model across a range of frequencies. The structural model is sufficiently accurate between 1 and $5 \mathrm{~Hz}$ so that by accounting for the unmodeled dynamics with an additive uncertainty model a control law can be synthesized which stabilizes the system and performs well when implemented. Development of better uncertainty models to describe the model errors can further increase the performance of control laws on the experimental flexible structure.

\subsection{Input Multiplicative Uncertainty}

A series of control laws is synthesized using additive uncertainty and input multiplicative uncertainty descriptions to account for variations in the model. The block diagram in figure 8 describes the problem formulation. The output uncertainty, sensu, is set to zero in this series of designs. Ten control laws are formulated for input multiplicative uncertainty level varying between 0 and $25 \%$. Robustness and performance of the control designs are traded off in the design process, as one is increased the other is decreased. An iterative procedure is preformed until a $\mu$ value of approximately 1 is achieved. This is done by selecting a desired level of input uncertainty and scaling the performance requirement, perfwt, until the the control design achieves a $\mu$ value of 1 . A control law with a $\mu$ value of 2.0 indicates that for the uncertainty and performance criteria prescribed, the control law achieves $\frac{1}{2}$ or $50 \%$ of the performance for $\frac{1}{2}$ or $50 \%$ of the uncertainty level.

Each control law is designed for a specific level of uncertainty, $\alpha$. For the purpose of this discussion, we will only refer to the input mul. tiplicative weight, actu, as the uncertainty parameter $\alpha$. The additive uncertainty is considered to be part of the design model since it held constant in each control design. For a prescribed level of uncertainty, $\alpha$, using the $\mu$-synthesis methodology, we are able to design a control law, $\bar{K}$, which achieves a performance level of $\beta$, corresponding to $\frac{1}{p e r / w t}$. This provides the point " $x$ " on the curve in figure 10 . A $\beta$ equal to 1 corresponds to the closed-loop performance equaling the open-loop performance, for $\beta$ less than 1, the closed-loop performance is better than the open-loop, and for $\beta$ greater than 1, performance is worse than the open-loop response. Assuming the system to be controlled is described exactly by the set of plants defined by the nominal model and uncertainty descriptions, the level of performance achieved for the worst case input signal affecting the worst case plant model can be formulated as an $H_{\infty}$ control probiem.

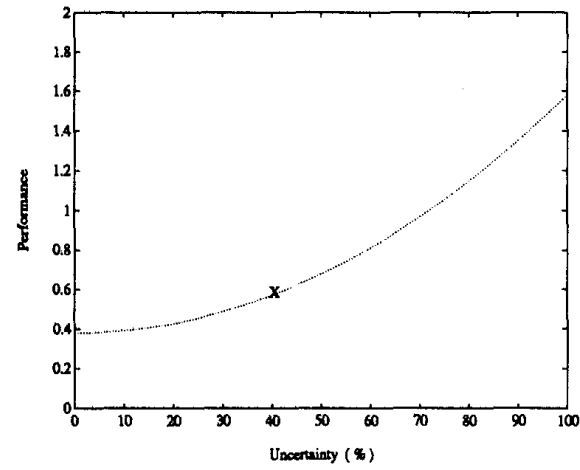

Figure 10: Control Design, $\bar{K}$, for an Uncertainty Level of $40 \%$

Suppose that the initial model set, which is described by the nominal structural model and uncertainty descriptions, is a conservative representation of the "real" structure. That is, extra plants are included in the model set which are not feasible. The control law $\bar{K}$ designed for this model set will likely achieve better performance when implemented on the "real" structure than is anticipated. The improved performance is due to the fact that the predicted performance level, $\beta$, is based on the vorst plant model in the initial model set. If the "real" system does not correspond to the worst case model in the set, the performance level of the closed-loop system will be higher than the design value.

Similarly, if the uncertainty description does not encompass the "real" system, the control law may destabilize the system or degrade performance severely. As the variation between the design model and the physical system increases, the performance of the control law, $\bar{K}$, degrades. This degradation corresponds to an increase in the performance norm. A graphical representation of this is presented in figure 10. The dotted line indicates how the performance, $\beta$, of the control law $\bar{K}$ might vary as a function of the uncertainty level $\alpha$. As an example, the control law $\bar{K}$ is designed for an uncertainty level of $40 \%$, and achieves a performance of 0.58 . If there is less uncertainty between the "real" system and the model, the control law will exhibit improved performance when implemented. Conversely, if there is more variation between the "real" system and model exists, the control law performance will degiade.

A number of control laws, $K_{1}$ to $K_{6}$, are designed for $3 \%, 10 \%$, $20 \%, 40 \%, 60 \%$, and $80 \%$ uncertainty, each generating a curve similar to the one in figure 10. A graph of these curves is shown in figure 11. Each " $x$ " in the figure corresponds to a $\mu$-synthesis controller synthesized for the specified level of uncertainty and the corresponding level of performance. Each control law would have a $\mu$ value of 1 . The solid curve represents the envelope of achievable performance for the control designs based on the nominal model and the uncertainty de scription. As one would expect, the highest performance is achieved when the nominal model is a perfect representation of the "real" system. Based on these graphs, one can see that accurately describing the physical system with non-conservative sets of plants results in a superior performing control law on the "real" system. This result is based 
on a given model and uncertainty descriptions. Changing the nominal model and/or uncertainty description can significantly alter the characteristics of this curve. Designing a series of controllers based on different uncertainty levels can be employed to verify the consistency of the model and uncertainty descriptions with experimental data.

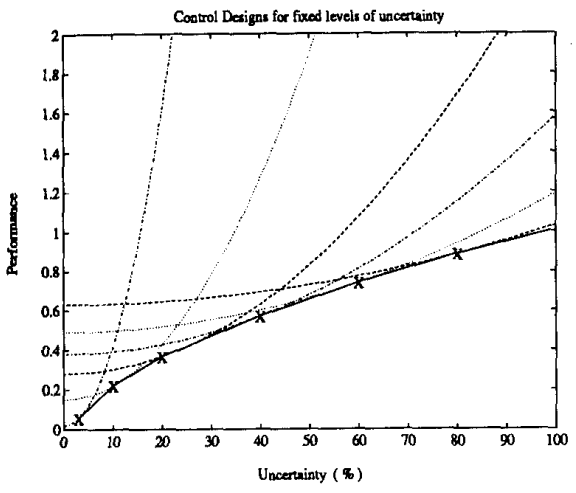

Figure 11: Six Control Designs for Uncertainty Levels of $3 \%, 10 \%$, $20 \%, 40 \%, 60 \%, 80 \%$

Ten controllers are synthesized for the Caltech structure based on a design model with different levels of input multiplicative uncertainty to examine robustness and performance tradeoffs. In each design, the performance weight perfut is scaled to achieve a $\mu$ value of 1 . A plot of $\mu$ across frequency for $K 1 \mathrm{am}, K 3 a \mathrm{~m}$, and $K 10 \mathrm{am}$ is shown in figure 15.

The value of $\mu$ is highest within the frequency range of the flexible modes to be controlled. Attenuation of these modes is the limiting factor in the controller design, which is often the case in lightly damped, flexible systems. This limitation results in the closed-loop system, with an $H_{\infty}$ optimal controller, not having a flat frequency response. The frequency peaks are associated with these modes limit the achievable $H_{\infty}$ norm in the control design problem.

The level of sensor noise, senswt, is fixed at $2 \times 10^{-3}$ in all the problem formulations. This represents the relative noise level measured experimentally. The actuator weights, magwt and ratewt, are selected to correspond to the magnitude and rate limits of the actuators. For these control designs, magwt is set to 80 and the ratewt is set to 3770 . Table 3 contains the parameters varied in the control designs.

\subsubsection{Experimental Results}

Ten controllers synthesized for the flexible structure experiment by varying the input multiplicative uncertainty level in the design model. These are implemented on the experiment and compared to the openloop response. Experimental data is derived from the filtered noise input to air actuator 1 and accelerometer 1,2 and 3. The open-loop frequency responses of accelerometers 1,2 and 3 are shown in figure 16 . The closed-loop experimental frequency responses of accelerometers 1 , 2 and 3 with controllers $K 3 \mathrm{am}$ and $K 10 \mathrm{am}$ implemented are shown in figures 18 and 19. Table 4 contains the raw experimental data of the closed-loop experiments for each control design. A singular value plot of the loop gain for $\mathrm{K3am}$ is provided in figure 17. Time histories of the open-loop and c'osed-loop response of control designs $K 3 \mathrm{am}$ and K10am are shown in figures 20,21 and 22 .

Performance on the experiment is measured as the ratio of the maximum closed-loop peak response to the maximum open-loop response. The maximum frequency response magnitude peaks corresponding to accelerometers 1,2 and 3 are given in table 4 . The ratio of the maximum peak of the closed-loop control law to the open-loop response corresponds to the experimental performance.

The best performance, 0.073 , representing an attenuation of the maximum frequency domain peak by a factor of 13.7, is achieved for the control law designed with $2.25 \%$ input uncertainty. Control laws

\begin{tabular}{|c|c|c|c|c|}
\hline $\begin{array}{c}\text { Control } \\
\text { Law }\end{array}$ & $\begin{array}{c}\text { Actuator } \\
\text { Uncertainty (\%) }\end{array}$ & per fwt & $\begin{array}{c}\text { Predicted } \\
\text { Performance }\end{array}$ & $\mu$ \\
\hline K1am & 0.00 & 13.0 & .077 & 1.02 \\
K2am & 1.00 & 12.4 & .081 & 1.02 \\
K3am & 2.25 & 11.0 & .091 & 1.01 \\
K4am & 4.00 & 10.0 & .100 & 1.02 \\
K5am & 7.29 & 8.4 & .119 & 1.03 \\
K6am & 10.00 & 7.1 & .141 & 1.00 \\
K $7 a m$ & 14.44 & 5.8 & .172 & 1.08 \\
K $8 a m$ & 17.00 & 4.2 & .238 & 1.07 \\
K9am & 20.25 & 3.9 & .256 & 1.02 \\
K10am & 25.00 & 2.9 & .345 & 1.02 \\
\hline
\end{tabular}

Table 3: Control Design Parameters for Input Uncertainty

\begin{tabular}{|c|c|c|c|c|c|}
\hline \multirow{2}{*}{$\begin{array}{l}\text { Control } \\
\text { Law }\end{array}$} & \multicolumn{3}{|c|}{$\begin{array}{c}\text { Maximum Magnitude in } \\
\text { Frequency Range } 0-10 \mathrm{~Hz} \\
\text { Raw Data }\end{array}$} & \multirow{2}{*}{$\begin{array}{l}\text { Experimental } \\
\text { Performance }\end{array}$} & \multirow{2}{*}{$\begin{array}{l}\text { Predicted } \\
\text { Performance }\end{array}$} \\
\hline & Sensor 1 & Sensor 2 & Sensor 3 & & \\
\hline Open-loop & 2.1511 & 1.2450 & 0.5232 & 1.0000 & 1.000 \\
\hline K1am & 0.187 & 0.139 & 0.176 & 0.087 & 0.077 \\
\hline K2am & 0.187 & 0.082 & 0.028 & 0.087 & 0.081 \\
\hline$K 3 a m$ & 0.157 & 0.074 & 0.024 & 0.073 & 0.091 \\
\hline K4am & 0.176 & 0.087 & 0.026 & 0.082 & 0.100 \\
\hline K5am & 0.200 & 0.088 & 0.025 & 0.093 & 0.119 \\
\hline K6am & 0.261 & 0.131 & 0.029 & 0.121 & 0.141 \\
\hline K7am & 0.306 & 0.124 & 0.026 & 0.142 & 0.172 \\
\hline K8am & 0.265 & 0.181 & 0.025 & 0.123 & 0.238 \\
\hline K9am & 0.223 & 0.160 & 0.020 & 0.104 & 0.256 \\
\hline $\mathrm{K} 10 \mathrm{am}$ & 0.346 & 0.247 & 0.030 & 0.161 & 0.345 \\
\hline
\end{tabular}

Table 4: Experimental Results of Input Uncertainty Designs

designed for higher and lower uncertainty levels than this exhibited reduced levels of performance. $K 1 \mathrm{am}$ and $\mathrm{K2am}$ achieved performance levels less than precicted by the design model, and all other control designs surpassed their predicted performance. Figure 12 is a plot of the designed performance level and the experimentally derived performance levels as a function of input multiplicative uncertainty level. Circles, ' $o$ ', represent the experimental values and ' $x$ ' represent the model.

One can interpret this graph as one interprets figure 11. The set of models described by the problem formulation for designs $K 1 a m$ and $K 2 a m$ do not encompass the "real" system, because for the worst case plant description the performance levels are higher than achieved when implemented on the experimental structure. Either models for these controllers can not ba validated or the "real" system does not lie inside this set of plant models. One can infer that the control designs are optimized for an inaccurate model. The set of plant models defined in the control design problems for $\mathrm{K3am}$ through $\mathrm{K10am}$ provide a good representation of the "real" system due to the experimental and performance levels corresponding to the designed performance level. Data can never valiciate a model because the next set of experiments may invalidate it. All that can be said about the model sets for control designs $K 3 \mathrm{am}$ through $K 10 \mathrm{am}$ is that they provide an accurate description of the physical system for control design.

Selecting an appropriate level of uncertainty, $2.25 \%$ for this problem description, provides the highest level of performance on the structure. Increasing the input uncertainty level leads to more conservative control laws which emphasize robustness. These control laws reduce the amount of control action leading to a reduction in the attenuation level. The selection of uncertainty descriptions has a direct bearing on the performance and robustness of the control designs.

\subsection{Output Multiplicative Uncertainty}

A set of control laws is synthesized with additive and output multiplicative uncertainties to account for errors in the design model. The problem formulation is based on the block diagram in figure 8 with the input uncertainty scaling, actu, set to zero. Nine control laws are formulated for the output scaling, sensu, varying between 0.1 and 0.5 . This is analogous to the output multiplicative uncertainty varying be- 
tween $1 \%$ and $25 \%$. Each control law is designed for a specified level of output uncertainty, sensu, with the performance weight, perfwt, scaled to achieve a $\mu$ value of 1 .

\begin{tabular}{|c|c|c|c|c|}
\hline $\begin{array}{c}\text { Control } \\
\text { Law }\end{array}$ & $\begin{array}{c}\text { Sensor } \\
\text { Uncertainty (\%) }\end{array}$ & perfwt & $\begin{array}{c}\text { Predicted } \\
\text { Performance }\end{array}$ & $\mu$ \\
\hline$K 1 \mathrm{sm}$ & 1.00 & 11.60 & .086 & 1.03 \\
$K 2 \mathrm{sm}$ & 2.25 & 10.95 & .091 & 0.95 \\
$K 3 \mathrm{sm}$ & 4.00 & 10.40 & .096 & 1.05 \\
$K 4 \mathrm{sm}$ & 7.29 & 9.70 & .103 & 1.03 \\
$K 5 \mathrm{sm}$ & 10.00 & 9.10 & .110 & 1.06 \\
$K 6 \mathrm{sm}$ & 14.44 & 8.80 & .114 & 1.03 \\
$K 7 \mathrm{sm}$ & 17.00 & 8.40 & .119 & 0.96 \\
$K 8 \mathrm{sm}$ & 20.25 & 8.10 & .124 & 1.07 \\
$K 9 \mathrm{sm}$ & 25.00 & 7.75 & .129 & 1.09 \\
\hline
\end{tabular}

Table 5: Control Design Parameters for Output Uncertainty

The set of nine control laws uses the same noise weight, senswt, magwt, and ratewt as in the set of input uncertainty designs. Table 5 contains a list of parameters varied in the output multiplicative uncertainty control designs. Each control law is implemented on the structure and an exf erimental frequency responses are generated from the air disturbance input at sensor 1 to the three accelerometer out puts. The closed-loop experimental frequency responses with $\mathrm{K} 3 \mathrm{sm}$ implemented are shown in figure 23. A time history of the closedloop response using $\mathrm{K} 3 \mathrm{sm}$ is shown in figure 24 . Table 6 contains the raw experimental data from the closed-loop experiments. $\mathrm{K} 1 \mathrm{am}$ is included because it was designed with zero input/output multiplicative uncertainty.

The best control design, $K 3 \mathrm{sm}$, achieved a performance level of 0.072. Klam had a performance level less than predicted and all other control laws exceeded their predicted performance. Figure 13 provides a comparison between the predicted performance of the model given the designed uncertainty level and the experimental data. Note the consistent trend in the data between the theory and the experiments. As expected, increasing the output uncertainty weight increases the robustness characteristics of the control law at the expense of the performance. The high correlation between the experimental and predicted performance levels indicate that the nominal model with output multiplicative uncertainty provides an excellent model of the experimental flexible structure for the purpose of control.

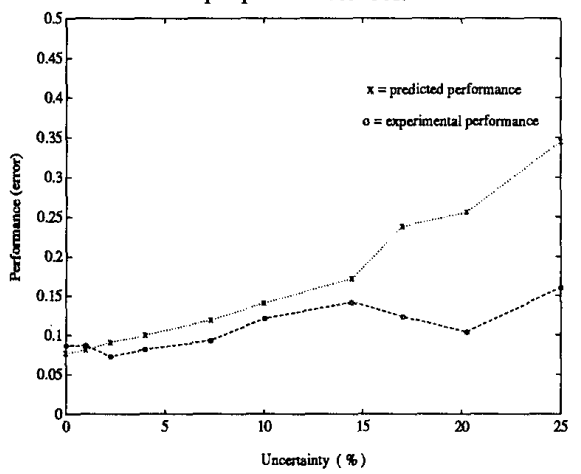

Figure 12: Predicted versus Experimental Performance for the Input Uncertainty Designs

\section{Summary}

Representing the physical system with a nominal model and an uncertainty description provides an excellent design model for use in the $\mu$-synthesis techniques. The addition of uncertainty models is required because the inclusion of sensor noise models alone will not provide the required robustness at desired locations in the plant. The series of control laws developed using input uncertainty reflect a strong dependence of the control laws on accurate input signals to the system. As the input uncertainty level is increased in the control design model, there is a marked decrease in the closed-loop performance. Control

\begin{tabular}{|c|c|c|c|c|c|}
\hline \multirow{2}{*}{$\begin{array}{c}\text { Control } \\
\text { Law }\end{array}$} & \multicolumn{3}{|c|}{$\begin{array}{c}\text { Maximum Magnitude in } \\
\text { Frequency Range } 0-10 \mathrm{~Hz} \\
\text { Raw Data }\end{array}$} & \multirow{2}{*}{$\begin{array}{c}\text { Experimental } \\
\text { Performanee } \\
\text { OL:Cl. }\end{array}$} & \multirow{2}{*}{$\begin{array}{l}\text { Predirched } \\
\text { Performatire } \\
\text { OL,:CI. }\end{array}$} \\
\hline & Sensor 1 & Sensor 2 & Sensor 3 & & \\
\hline Open-loop & 2.151 & 1.245 & $0 . \overline{523}$ & 1.0000 & 1.00 \\
\hline K1am & 0.187 & 0.139 & 0.176 & 0.087 & 0.077 \\
\hline $\mathrm{K} 2 \mathrm{sm}$ & 0.182 & 0.152 & 0.182 & 0.085 & $0.0 \times 6$ \\
\hline K3sm & 0.154 & 0.123 & 0.138 & 0.072 & 0.091 \\
\hline$K 4 s m$ & 0.177 & 0.142 & 0.140 & 0.082 & 0.096 \\
\hline$K 5 \mathrm{sm}$ & 0.180 & 0.138 & 0.127 & 0.084 & 0.103 \\
\hline$K 6 \mathrm{sm}$ & 0.195 & 0.145 & 0.131 & 0.091 & 0.110 \\
\hline $\mathrm{K} 7 \mathrm{sm}$ & 0.180 & 0.122 & 0.113 & 0.084 & 0.114 \\
\hline$K 8 s m$ & 0.185 & 0.105 & 0.105 & 0.086 & 0.119 \\
\hline$K 9 \mathrm{sm}$ & 0.208 & 0.124 & 0.047 & 0.097 & 0.124 \\
\hline $\mathrm{K} 10 \mathrm{sm}$ & 0.229 & 0.131 & 0.054 & 0.106 & 0.129 \\
\hline
\end{tabular}

Table 6: Experinental Results of Output Uncertainty Designs

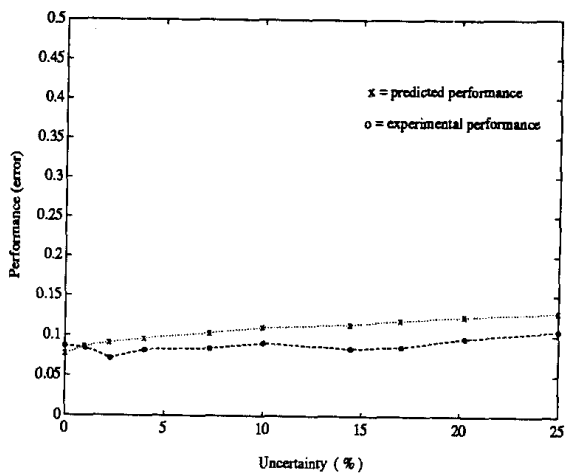

Figure 13: Predicted versus Experimental Performance for Output Uncertainty Designs

designs for the phase I flexible structure are less sensitive to output uncertainty, which provides a very accurate description of the system when combined with the nominal model for control design. The output multiplicative contrcl designs exhibit better performance both theoretically and experimentally as a function of uncertainty. The theoretical and experimental results indicate that uncertainty modeling plays a major role in the tradeoff of performance requirements and robustness properties of synthesized control laws. Therefore, accurate accounting of model error and its location in the problem formulation should be incorporated into the system identification process.

A number of improvements can be made in the methods for modeling and identification of flexible structures for control design. Identification methods should produce nominal models with both perturbations and additive noise for incorporation into the control problem formulation. These identified models should then be used to improve the first principles model of the structure. Incorporation of control design, identification and modeling into a more integrated framework is necessary to achieve the stringent performance requirements for seen on flexible structures.

\section{References}

[Balas] G.J. Balas, "Robust Control of Flexible Structures: Theory and Experiments," Ph.D. Thesis, California Institute of Technology, Pasadena, 1989.

[BalChuD] G.J. Balas, C.C. Chu and J.C. Doyle, "Vibration Damping and Robust Control of the JPL/AFAL Experiment Using $\mu$-Synthesis," Proceedings of $28^{\text {th }}$ Conference on Decision and Control, Ft. Lauderdale, FL, December, 1989.

[BalDoy1] G.J. Balas and J.C. Doyle, "Identification for robust control of flexible structures," ACC, Pittsburgh, PA, June, 1989.

[BalDoy2] G.J. Balas and J.C. Doyle, "Robust Control of Flexible Modes in the Controller Crossover Region," ACC89, Pittsburgh, PA, 1989. 
[DGKF] J.C. Doyle, K. Glover, P. Khargonekar and B.A. Francis, "State-space solutions to standard $H_{2}$ and $H_{\infty}$ control problems," IEEE Transactions on Automatic Control, Vol. 34, No. 8, August, 1989, pp 831-847.

[Doy1] J.C. Doyle, "Analysis of feedback systems with structured uncertainties," Proc. IEE-D 129, 1982, pp 242-250.

[Doy2] J.C. Doyle, Lecture notes on advances in multivariable control,

ONR/Honeywell Workshop on Advances in Multivariable Control, Minneapolis, MN, October, 1984.

[DoyPL] J.C. Doyle, K. Lenz and A. Packard, "Design Examples using $\mu$-synthesis: Space Shuttle Lateral Axis FCS during reentry," IEEE CDC, December 1986, pp. 2218-2223.

[Francis] B.A. Francis, A Course in $H_{\infty}$ Control Theory, SpringerVerlag, Berlin, 1987.

[GlovDoy1] K. Glover and J.C. Doyle, "State-space formulae for all stabilizing controllers that satisfy an $H_{\infty}$-norm bound and relations to risk sensitivity," Systems \& Control Letters 11, 1989, pp $167-172$

[GlovDoy2] K. Glover and J.C. Doyle, "A state-space approach to $H_{\infty}$ optimal coatrol," to appear in Systems \& Control Letters.

[Pack] A.K. Packard, "What's new with $\mu$ : structured uncertainty in multivariable control," Ph.D. Thesis, University of California at Berkeley, 1988.

[MUSYN] MUSYN Robust Control Short Course Lecture Notes, Arcadia, CA, September, 1989.

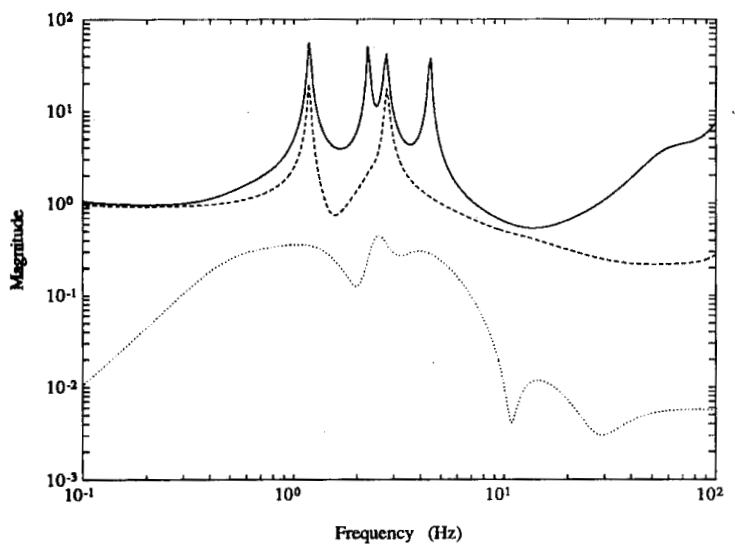

Figure 14: Singular Value Plot of the Loop Gain for Controller $K 1 s n$

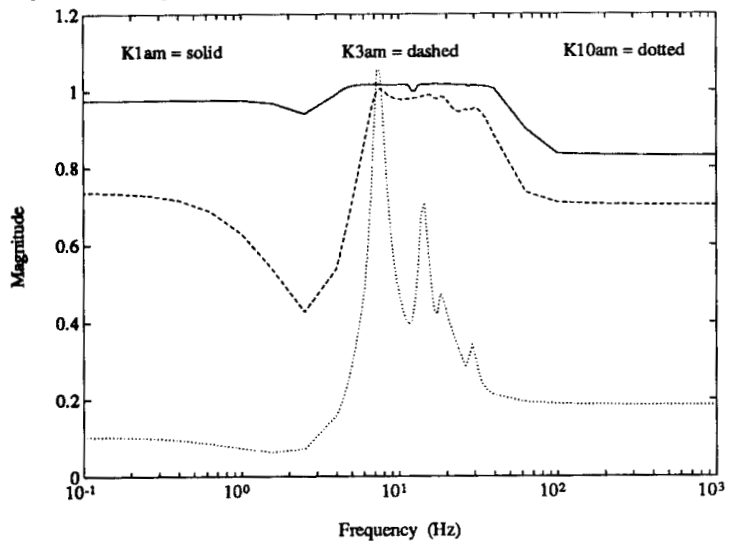

Figure 15: A Plot of $\mu$ for Control Designs $K 1 a m, K 3 a m$ and $K 10 a m$

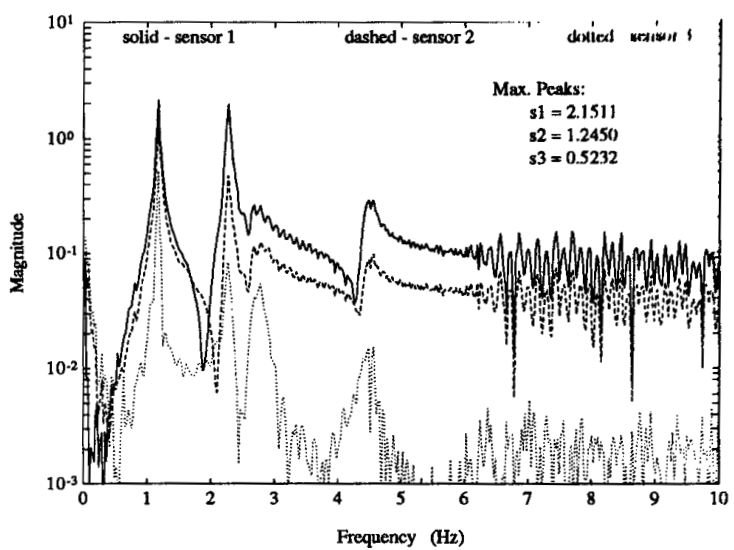

Figure 16: Open-loop Response of Sensors to 1-6 Hz Sine Sweep into Air 1

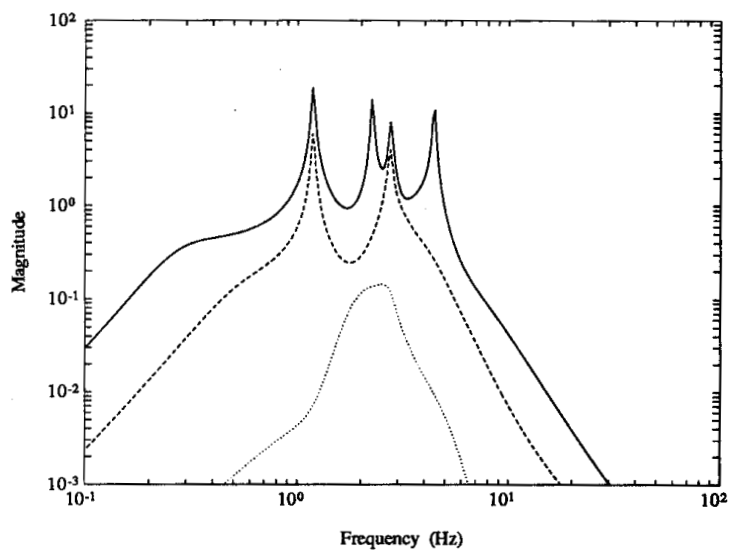

Figure 17: Singular Value Plot of the Loop Gain for Controller $\mathrm{K} 3 \mathrm{am}$

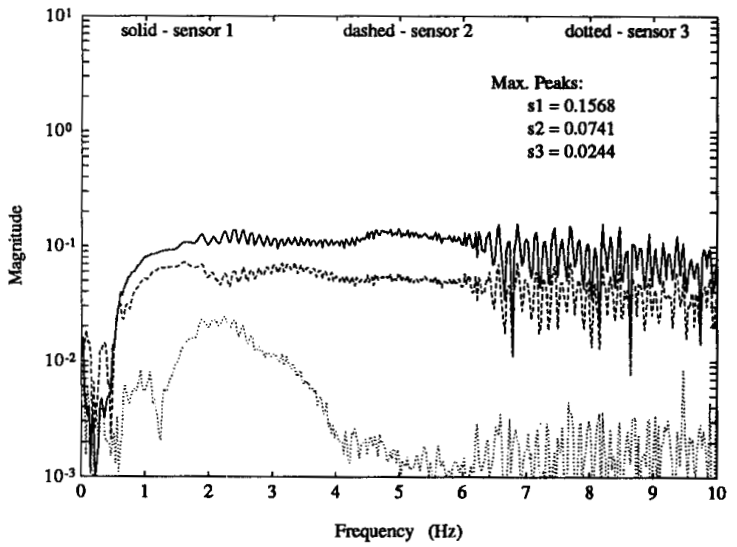

Figure 18: Closed-loop Response, $\mathrm{K} 3 a m$, of Sensors to $1-6 \mathrm{~Hz}$ Sine Sweep into Air 1 


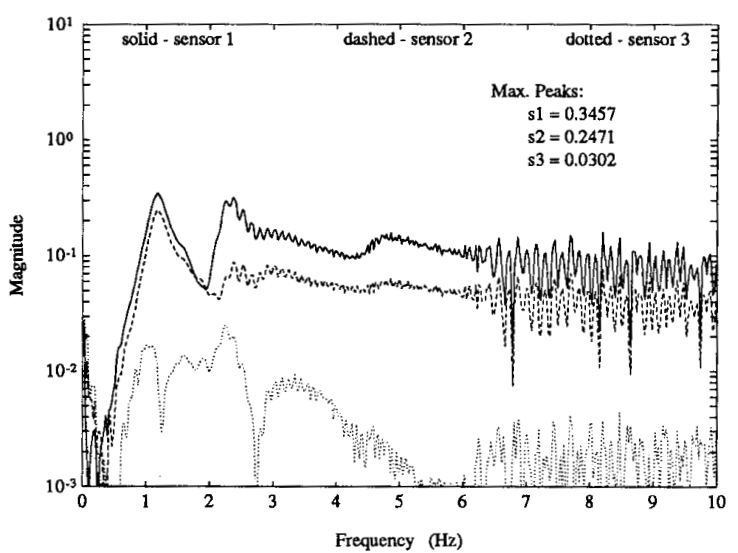

Figure 19: Closed-loop Response, $K 10 \mathrm{am}$, of Sensors to 1-6 Hz Sine Sweep into Air 1

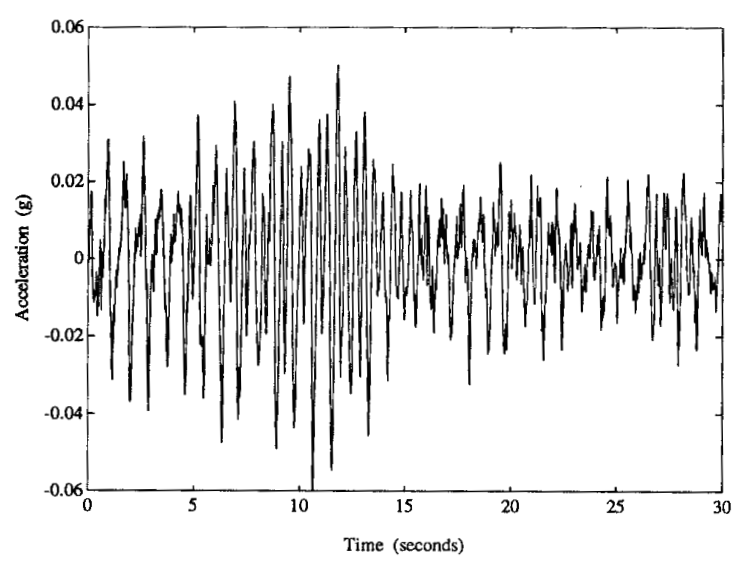

Figure 20: Open-loop Sensor 1 Response to 1-6 Hz Sine Sweep into Air Actuator 1

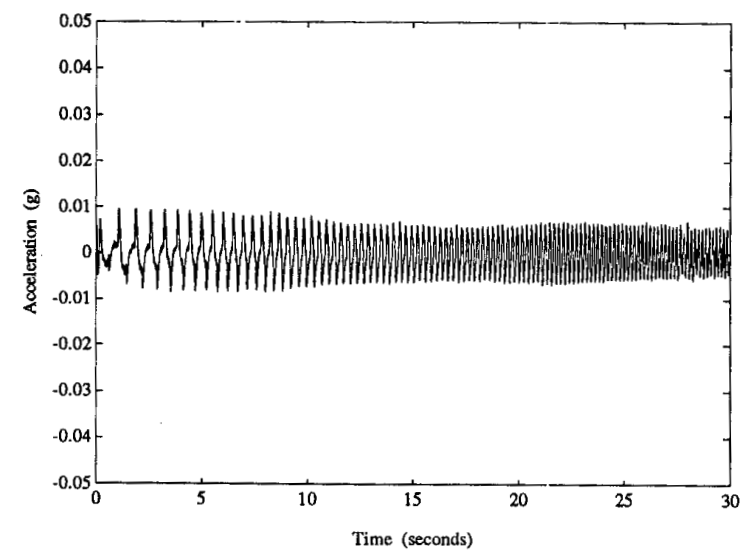

Figure 21: Closed-loop Time Response, $\mathrm{K3am}$, of Sensor 1 to 1-6 $\mathrm{Hz}$ Sine Sweep into Air 1

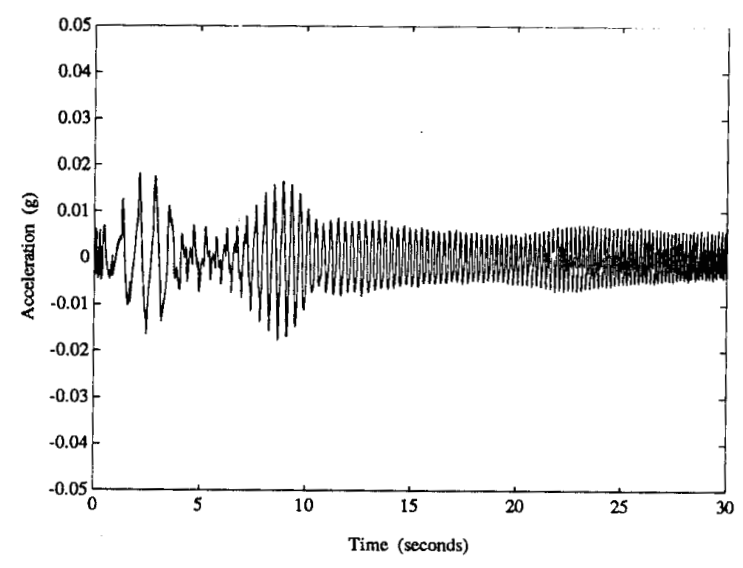

Figure 22: Closed-loop Time Response, $K 10 \mathrm{am}$, of Sensor 1 to 1-6 $\mathrm{Hz}$ Sine Sweep into Air 1

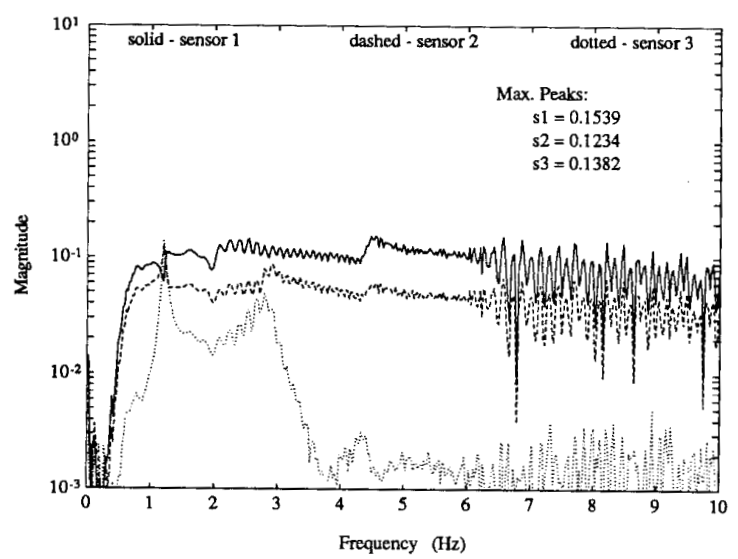

Figure 23: Closed-lcop Response, $\mathrm{K3sm}$, of Sensors to 1-6 $\mathrm{Hz}$ Sine Sweep into Air 1

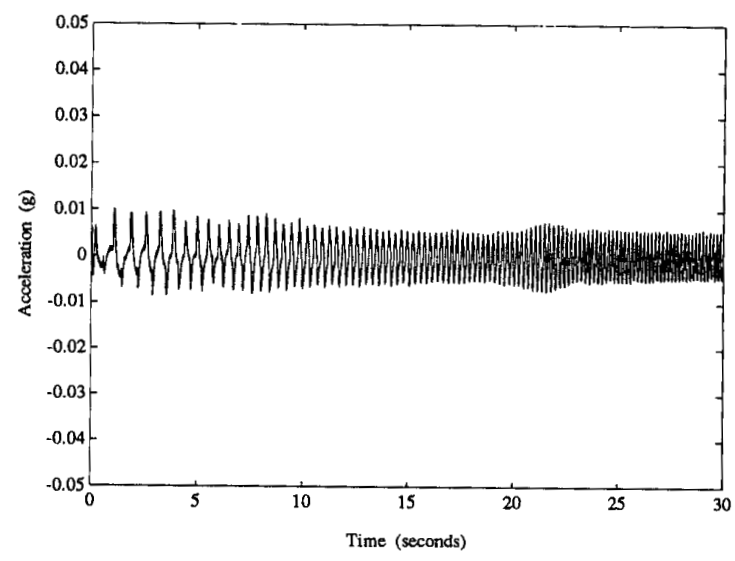

Figure 24: Closed-loop Time Response, $K 3 s m$, of Serisor 1 to $1-6 \mathrm{~Hz}$ Sine Sweep into Air 